\title{
Applying Local Cooccurring Patterns for Object Detection from Aerial Images
}

\author{
Wenjing Jia ${ }^{1}$, David Tien ${ }^{2}$, Xiangjian $\mathrm{He}^{1}$, Brian A. Hope ${ }^{3}$, and Qiang $\mathrm{Wu}^{1}$ \\ 1 Faculty of Information Technology, University of Technology, Sydney \\ PO Box 123, Broadway, NSW 2007, Australia \\ $\{$ wejia, sean, wuq\}@it.uts.edu.au \\ 2 School of Information Technology, Charles Sturt University, \\ Panorama Avenue Bathurst, NSW, 2795, Australia \\ dtien@csu.edu.au \\ 3 Department of Lands, NSW, Australia \\ Tony .Hope@lands.nsw.gov . au
}

\begin{abstract}
Developing a spatial searching tool to enhance the search capabilities of large spatial repositories for Geographical Information System (GIS) update has attracted more and more attention. Typically, objects to be detected are represented by many local features or local parts. Testing images are processed by extracting local features which are then matched with the object's model image. Most existing work that uses local features assumes that each of the local features is independent to each other. However, in many cases, this is not true. In this paper, a method of applying the local cooccurring patterns to disclose the cooccurring relationships between local features for object detection is presented. Features including colour features and edge-based shape features of the interested object are collected. To reveal the cooccurring patterns among multiple local features, a colour cooccurrence histogram is constructed and used to search objects of interest from target images. The method is demonstrated in detecting swimming pools from aerial images. Our experimental results show the feasibility of using this method for effectively reducing the labour work in finding man-made objects of interest from aerial images.
\end{abstract}

Key words: Local cooccurring patterns, colour cooccurrence histogram, swimming pool detection

\section{Introduction}

Developing a spatial searching engine to enhance the search capabilities of large spatial repositories for Geographical Information System (GIS) update, which is able to automate the process of identifying objects from high-resolution aerial images and in turn update the vector data, has now attracted more and more attention of researchers from both the area of data mining and the areas of image analysis and pattern recognition. One of the typical goals of such searching engine is to automatically search the required image for an object and return 
a list of possible matches for that item. Content-based image retrieval (CBIR) techniques which combine both spatial and textual features of images have been widely used. In a typical spatial searching engine system, users specify one of the objects they are looking for as a basis of the searching; the system searches the required image for the object and returns a list of possible matches for that item. The positions of the matched objects can then be used to update the vector data of GIS system $[1,2]$. The recent interest is mainly in identifying objects from high-resolution aerial images, thanks to the technical development on highresolution remote sensor. The major interest for such purpose is in identifying small structures, such as individual road segments and buildings. Objects that need to be identified include various man-made buildings, housing, properties, swimming pools, lakes, dams, roads, and vegetation.

Recognition of visual objects is a fundamental task in vision. Typically, objects to be recognized are represented by many local features or local parts. Testing images are processed by extracting local features which are then matched with the object's model image. Most existing work that uses local features assumes that each of the local features is independent to each other. However, in many cases, this is not true. For instance, the idea presented in [3], [4], and [5], uses the colour-based local features only. Hence, it is found to be sensitive to the selection of the training set and may lead to detection failures when there are other areas which have similar colour distribution or when the objects have larger colour variance with the training set. Many existing methods for building extraction use the shape information only. Hence, it is hard to differentiate them from other similar objects which apparently have different colours and edge distributions from the target. For our system, we propose applying the cooccurring patterns of multiple local features, such as colour distribution and edge-based shape features, for object detection [6].

In this paper, a way of disclosing the cooccurring relationships between local features such as colour and edge is demonstrated in swimming pool detection. The local cooccurring pattern (LCP), which consists of characteristic local features and the statistical cooccurrence relationship between them, is then applied to recognise and locate swimming pools from aerial images. Note that the objects of interest in this research, i.e., the swimming pools, may have various shapes and large variance in their colours values. To utilise the local coccurring patterns robustly, we introduce a histogram-based object detection method together with a robust histogram matching algorithm. A histogram representation, called colour cooccurrence histogram $(\mathrm{CCH})$, is introduced and utilised as the key to search through the input image and retrieve the regions of interest (ROIs), i.e., regions that have similar CCHs with the model histogram. During detecting process, a scanning window moves through the input image. At each position, the $\mathrm{CCH}$ histogram of the region covered by the scanning window will be calculated and compared with the model $\mathrm{CCH}$ histogram. Regions that have a higher matching score with the model $\mathrm{CCH}$ will be kept as the regions of interest (ROIs). Regions with low matching scores will be marked as background. To further refine the detection result, regions segmented by an edge-preserving 
image smoothing technique that matches with the retrieved ROIs are returned as the final detection regions.

The remaining parts of this paper are organised as follows. Section 2 first introduces the edge-preserving image smoothing procedure that are used to segment the input image. Then, colour cooccurrence histogram $(\mathrm{CCH})$, which reveals the coccurring patterns of colour and shape features in a simple way, are constructed and compared in Section 3 in order to find regions of interest. Experimental result of detecting swimming pool from aerial images is presented in Section 4. This paper is concluded in Section 5. Our future plan to continue this research is also mentioned in this section.

\section{Image Segmentation based on Edge-Preserving Smoothing}

Before extracting colour-based features, it is common that images are smoothed to obtain a smaller colour map and finer edge map. This can be achieved via two major ways: reducing the number of colours and filtering the input images.

The operation for reducing colour number is also called colour quantisation in references. In [7], colour quantisation was simply performed in RGB colour space using a $k$-means nearest neighbour algorithm. However, the RGB colour space is known to be sensitive to colour variation. In [8], an optimal colour quantisation algorithm based on variance minimisation in CIE Luv space [9] is employed by introducing only slight colour distortion into the quantised images. However, unlike the case mentioned in [8] where the colours of interested objects, vehicle number plates, only take a small number of colours, the colours of objects in an aerial image can be very large. Hence, using fixed number of colours to quantise an aerial image will unavoidly introduce large perceptible difference in quantised images for those small objects, which usually is the interest for many GIS applications. In [10], a perceptual colour naming method was employed in order to handle colour variation. The standard ISCC-NBS Colour Names Dictionary [11] was employed to quantise and name the colour space. Each pixel is assigned to the standard name of the closest ISCC-NBS centroid colour, according to the Euclidean distance in the CIE Lab colour space [12]. This method uses a single colour set for all images. Since it is needed to find a best match in the standard colour dictionary for each colour represented in the aerial image, it usually takes extremely long time to complete the task.

On the other hand, filtering, or smoothing, image is perhaps the most fundamental operation of image processing and computer vision. In the broadest sense of the term "filtering", the value of the filtered image at a given location is a function of the values of the input image in a small neighborhood of the same location [13]. This idea indiscriminately blurs the image, removing not only noise but also salient information. As edge-based shape features are equally important as colour features in our project, we need to preserve edge information as much as possible. Discontinuity-preserving smoothing techniques adaptively reduce the amount of smoothing near abrupt changes in the local structure, i.e., 
edges. In order to smooth the input image while keeping important edge information as much as possible to enable the extraction of both the color and edge based local features, we study the two most popular edge preserving smoothing techniques: bilateral filtering and mean shift filtering.

The bilateral filtering was introduced by Tomasi and Manduchi in [13] in 1998. Recently, with the extensive usage of the bilateral filtering in other areas than image denoising, such as demosaicking, image abstraction, image retinex, optical flow estimation, etc, a fast algorithm which uses signal processing approach to approximate the standard bilateral filter has been proposed by Paris and Durand in [14].

The basic idea underlying bilateral filtering is to do in the range domain of an image what traditional filters do in its spatial domain. Two pixels can be close to each other, that is, occupy nearby spatial location, or they can be similar to one another, that is, have similar values, possibly in a perceptually meaningful fashion [13].

Bilateral filtering can be achieved via a convolution of the image brightness function $f$ with a spatial filter $k_{s}$ and a range filter $k_{r}$ in a small area (called convolution window) surrounding each reference pixel of which the radius is determined by the spatial bandwidth $\sigma_{s}$. It will take a long time to carry on the convolution processing if the convolution window is large.

Another edge-preserving smoothing technique which is also based on the same principle, i.e., the simultaneous processing of both spatial and range domains, is mean shift filtering, proposed by Comaniciu and Meer in [15]. It has been noticed that the bilateral filtering uses a static window in both domains. The mean shift window is dynamic, moving in the direction of the maximum increase in the density gradient. Therefore, the mean shift filtering has a more powerful adaptation to the local structure of the data. In our algorithm, an image segmentation method based on mean shift filtering technique is used to obtain a finer detection result. The basic procedure of this method is illustrated as follows. For more details, please refer to [15].

Let $\left\{x_{i}\right\}_{i=1,2, \ldots, n}$ be the original image points, $\left\{z_{i}\right\}_{i=1,2, \ldots, n}$ be the points of convergence, and $\left\{L_{i}\right\}_{i=1,2, \ldots}$ be a set of labels indicating different segmented regions.

1. For each image point $\left\{x_{i}\right\}_{i=1,2, \ldots, n}$, run the mean shift filtering procedure until convergence and store the convergence point in $z_{i}=y_{i, c}$, as shown below:

(a) For each image point $\left\{x_{i}\right\}_{i=1,2, \ldots, n}$, initialise $j=1$ and $y_{i, 1}=x_{i}$. The first subscript $i$ of $y_{i, j}$ denotes the $i$ th image point, and the second subscript $j$ denotes the $j$ th iteration.

(b) Compute $y_{i, j+1}$ according to Equation 1 until convergence of $y_{i, c}$.

$$
y_{i, j+1}=\frac{\sum_{i=1}^{n} x_{i} g\left(\left\|\frac{x-x_{i}}{h}\right\|^{2}\right)}{\sum_{i=1}^{n} g\left(\left\|\frac{x-x_{i}}{h}\right\|^{2}\right)}
$$


(c) Assign $z_{i}=\left(x_{i}^{s}, y_{i, c}^{r}\right)$, which specifies the filtered data $z_{i}$ at the spatial location of $x_{i}^{s}$ to have the range components of the point of convergence $y_{i, c}^{r}$.

2. Delineate the clusters (suppose there are $m$ clusters), denoted by $\left\{C_{p}\right\}_{p=1,2, \ldots, m}$, in the joint domain by grouping together all $z_{i}$ which are closer than $h_{s}$ in the spatial domain and $h_{r}$ in the range domain under a Euclidean metric, i.e., concatenate the basins of attraction of the corresponding convergence points.

3. For each $i=1,2, \ldots, n$, assign $L_{i}=\left\{p \mid z_{i} \in C_{p}\right\}$.

Thus, using the mean shift procedure in the joint spatial-range domain, the input colour aerial images are segmented into many regions, each of them are represented with different colours. Parameters $h_{r}$ and $h_{s}$ are set experimentally.

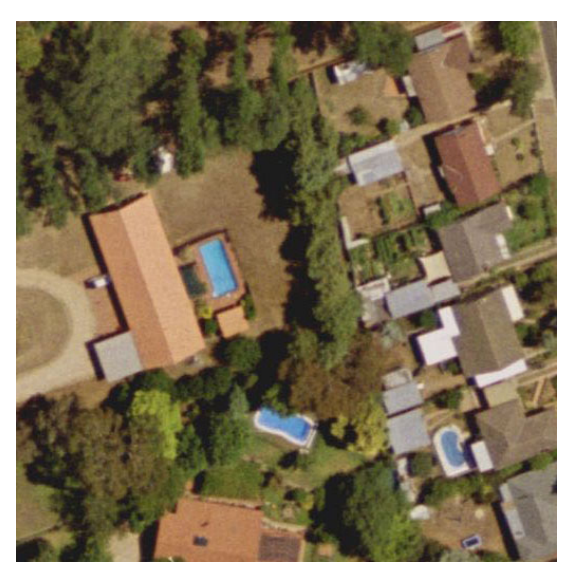

(a) Original image

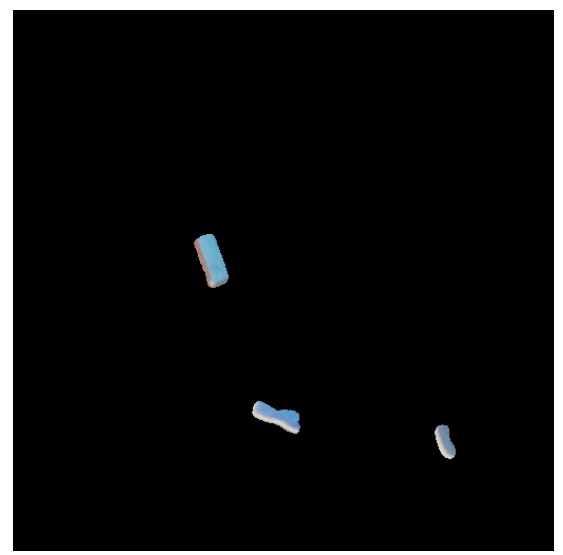

(c) Detected ROIs

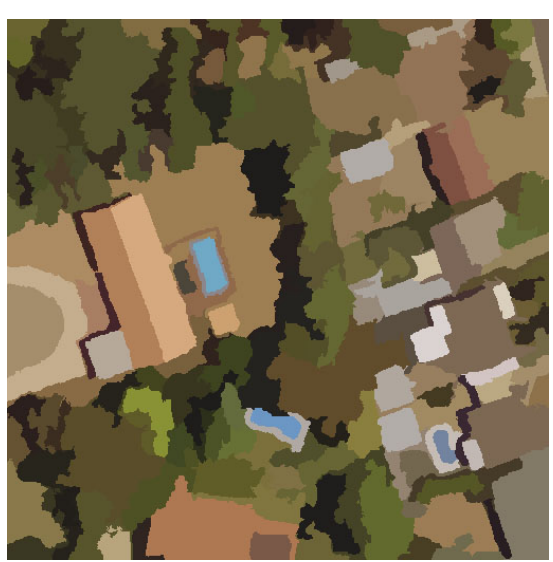

(b) Mean shift segmented

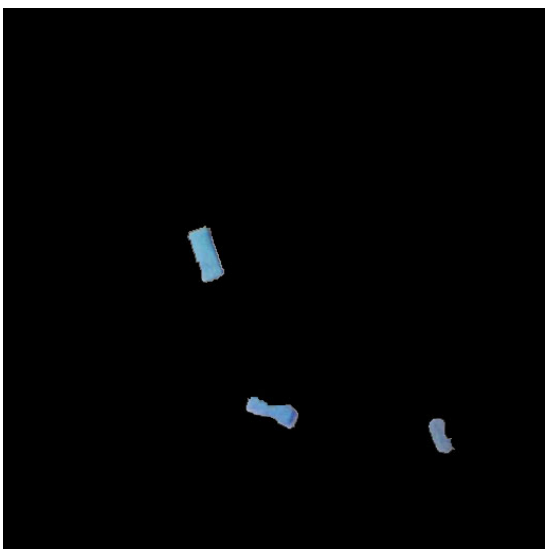

(d) Final detection result

Fig. 1. The procedure of the algorithm. 
Figure 1(a) shows an example of aerial image. Figures 1(b) shows the segmented image using a mean shift filter $\left(\left(\sigma_{s}, \sigma_{r}\right)=(7,5)\right)$. As it can be seen from Figure 1(b), applying the edge-preserving smoothing techniques, image noise appearing in the original image has been smoothed out, and the colours of objects in the image becomes much more uniform. This may lead to a better edge detection result, which in turn leads to a better shape representation, and simpler colour-based feature representation. On the other hand, if we look at the edges, such as the road boundary or the buildings' boundaries, the edges are very well kept and become sharper.

\section{Detecting ROIs Using Colour Cooccurrence Histogram}

\subsection{Constructing Colour Cooccurrence Histogram (CCH)}

Each element of the $\mathrm{CCH}$ histogram of a 2-D colour image $f(x, y)$ records the frequencies of occurrence of pairs of pixels that are at a distance away and take specified colour values.

Let us denote the colour value at point $p_{1}$ as $\boldsymbol{c}_{1}$, and the colour value at point $p_{2}$ as $\boldsymbol{c}_{2}$. The corresponding bin of the CCH histogram, denoted by $h\left(\boldsymbol{c}_{1}, \boldsymbol{c}_{2}, l\right)$, is counted as:

$$
h\left(\boldsymbol{c}_{1}, \boldsymbol{c}_{2}, l\right)=\operatorname{size}\left(\left\{\begin{array}{l|l}
\left(p_{1}, p_{2}\right) & \begin{array}{c}
p_{1}, p_{2} \in f, \text { and } \\
\left\|p_{1}-p_{2}\right\|=l
\end{array}
\end{array}\right\}\right), \boldsymbol{c}_{1}, \boldsymbol{c}_{2} \in \mathbf{C},
$$

where $\mathbf{C}$ is the colour set of the image, and the function size counts the number of elements in a set. In our experiments, the $l$ is set as 4 and $\left\|p_{1}-p_{2}\right\|$ uses Euclidean distance.

All image points in the whole image plane are scanned. The frequency of occurrence of the same colour pairs in the image at a distance away is recorded and summed in the corresponding bin of the $\mathrm{CCH}$ histogram.

Finally, the CCH histogram is normalised using the total number of points in the image.

\subsection{Comparing $\mathrm{CCH}$ Histograms}

After the $\mathrm{CCH}$ histogram is constructed, the next problem is how to compare two $\mathrm{CCH}$ histograms.

Normalised $L_{1}$-norm distance and the histogram intersection have been proposed in the past for comparing histograms. However, both metrics demand an identical colour matching in an exact bin-to-bin way between two histograms and thus are very sensitive to colour variance. In practice, the colours of real world images of same kind of objects can be varied both in the scene itself and in the image capturing process. This can be seen from the swimming pool images used in this research. Some examples are shown in Figure 2. Hence, images with same visual information but with different colour intensities may degrade the similarity level significantly when the conventional HI is used. 


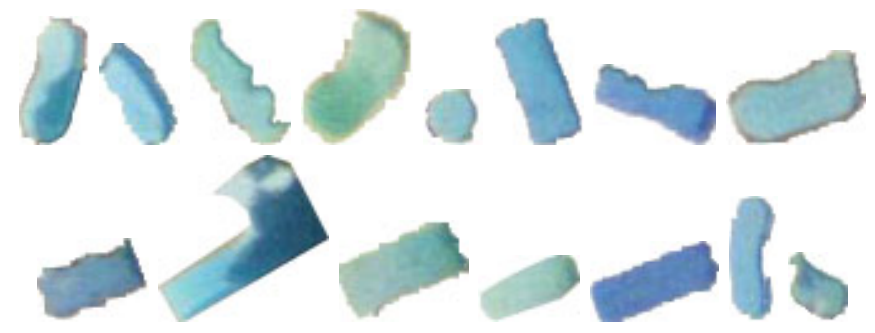

Fig. 2. Some examples of swimming pool images in our experiments.

In order to overcome this problem, Wong et al. [16] proposed a mergedpalette histogram matching (MPHM) method. The essence of the method is to extend the intersection from bins of identical colours to bins of similar colours. In their algorithm, as long as the distance between two colours is less than a fixed threshold, say $T h$, the intersection between the bins of these two colours will be calculated. This algorithm has produced more robust image retrieval results for images captured under various illumination conditions. However, it assumes an identical weight of the contribution between colours which have different similarities with the given colour. When applied to swimming pool images, the matching scores exhibited to be very sensitive to colour variations.

In [17], a Gaussian weighted histogram intersection (GWHI) method has been proposed which overcomes the above problems and has demonstrated to be much less sensitive to colour variations compared with the conventional HI method and Wong et al.'s MPHM method [17]. In this method, instead of applying identical weights to different colours, a weight function of colour distance has been employed to differentiate the matching between colours which have different distances to the given colour.

The GWHI method has shown to be less sensitive to colour variations. However, this method only addresses the intersecting of histogram bins between two colours. For the purpose of comparing two $\mathrm{CCH}$ histograms, where there are two pairs of colours involved in the intersection, the GWHI method is further extended to facilitate intersection of two pairs of colours.

In the following two subsections, the principles of the GWHI method is first introduced. Then, how to use the extended GWHI method to compare $\mathrm{CCH}$ histograms is described.

\subsection{Gaussian Weighted Histogram Intersection (GWHI)}

Assume all colours of pixels in the model image are taken from a colour set, denoted by $\mathbf{C}_{M}$, and all colours of pixels in the target image are taken from another colour set, denoted by $\mathbf{C}_{T}$ (the subscripts " $\mathrm{M}$ " and "T" denote for "model" and "target" respectively). Note that, the colour sets of two images can be either identical or different.

In [17], Jia et al. proposed applying a Gaussian weight function of colour distance to the conventional HI method in order to describe the relationship 
between the colour distance and the weight as:

$$
\eta=\sum_{\boldsymbol{c}_{i} \in \mathbf{C}_{M}} \sum_{\boldsymbol{c}_{j} \in \mathbf{C}_{T}} \min \left(h_{M}\left(\boldsymbol{c}_{i}\right), h_{T}\left(\boldsymbol{c}_{j}\right)\right) \cdot \exp \left(-\frac{\left\|\boldsymbol{c}_{i}-\boldsymbol{c}_{j}\right\|^{2}}{2 \sigma^{2}}\right), \quad\left\|\boldsymbol{c}_{i}-\boldsymbol{c}_{j}\right\| \leq B W
$$

where $h_{M}(\cdot)$ and $h_{T}(\cdot)$ are each element in the model histogram $H_{M}$ and the target histogram $H_{T}$. The parameter $\sigma$ and the bandwidth $B W$ in this equation can be selected empirically. In order to be consistent with Wong et al.'s MPHM algorithm, the contributions of identical colours in two methods are set as the same, and the overall weights in two methods are approximately the same. 99.9\% energy of Gaussian function is used to approximate its total energy in infinite space. It has been derived that $\sigma \doteq 0.8 T h$ and $B W \doteq 3.3 \sigma \doteq 2.6 T h$ [17] [18], where $T h$ is the threshold used in [16], as shown in Figure 3. Note that, from the below Equation 4 , it can be seen that $\left\|\boldsymbol{c}_{1}-\boldsymbol{c}_{2}\right\|$ never takes negative values. However, for an intuitionistic understanding, in this figure, weight functions when $\left\|\boldsymbol{c}_{1}-\boldsymbol{c}_{2}\right\|<0$ are also plotted.

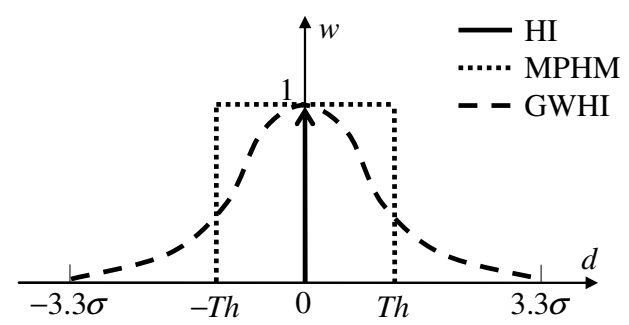

Fig. 3. Weight functions in three histogram intersection methods, where $d=\left\|\boldsymbol{c}_{1}-\boldsymbol{c}_{2}\right\|$.

The colour distance between two colours is defined as the perceptual difference in CIE Luv colour space as:

$$
\left\|\boldsymbol{c}_{1}-\boldsymbol{c}_{2}\right\|=\sqrt{\left(l_{1}-l_{2}\right)^{2}+\left(u_{1}-u_{2}\right)^{2}+\left(v_{1}-v_{2}\right)^{2}}
$$

where $\boldsymbol{c}_{1}=\left(l_{1}, u_{1}, v_{1}\right)$ and $\boldsymbol{c}_{2}=\left(l_{2}, u_{2}, v_{2}\right)$ are two colours represented in CIE Luv colour space. $l$ takes a value in the range of $[0,100], u$ takes a value in the range of $[-83,175]$, and $v$ takes a value from $[-134,107]$. The bandwidth in our experiments is set as $T h=15$, which is same as in [16]. Thus, we have $\sigma \doteq 16$ and $B W \doteq 52.8$.

\subsection{Comparing CCH Histograms Using Extended GWHI}

In order to compare two $\mathrm{CCH}$ histograms in perceptually similar colour bins, the following operations are performed.

Initialise the matching ratio $\eta$ as 0 . For each element $h_{M}\left(\boldsymbol{c}_{i}, \boldsymbol{c}_{j}, l\right)_{\mid \boldsymbol{c}_{i}, \boldsymbol{c}_{j} \in \mathbf{C}_{M}}$ in the CCH histogram of model image, scan each entry $h_{T}\left(\boldsymbol{c}_{m}, \boldsymbol{c}_{n}, l\right)_{\mid \boldsymbol{c}_{m}, \boldsymbol{c}_{n} \in \mathbf{C}_{T}}$ 
in the $\mathrm{CCH}$ histogram of target image and perform the following operations (1) through (3) until all entries in the model $\mathrm{CCH}$ histogram has been scanned:

1. Compute the colour distances $d_{1}=\left\|\boldsymbol{c}_{i}-\boldsymbol{c}_{m}\right\|$ between the first pair of colours $\boldsymbol{c}_{i}$ and $\boldsymbol{c}_{m}$, and $d_{2}=\left\|\boldsymbol{c}_{j}-\boldsymbol{c}_{n}\right\|$ between the second pair of colours $\boldsymbol{c}_{j}$ and $\boldsymbol{c}_{n}$, as defined in Equation 4. Take the larger distance as the distance between two pairs of colours, denoted by $d$, i.e., $d=\max \left(d_{1}, d_{2}\right)$.

2. If $d \leq B W$, where $B W$ is the bandwidth of Gaussian weight function (see Equation 3), decide the intersected section between two $\mathrm{CCH}$ histograms and apply the Gaussian weight function of distance as:

$$
\gamma=\min \left(h_{M}\left(\boldsymbol{c}_{i}, \boldsymbol{c}_{j}, l\right), h_{T}\left(\boldsymbol{c}_{m}, \boldsymbol{c}_{n}, l\right)\right) \times \exp \left(-\frac{d^{2}}{2 \sigma^{2}}\right), \quad d \leq B W,
$$

where $\boldsymbol{c}_{i}, \boldsymbol{c}_{j} \in \mathbf{C}_{M}$, and $\boldsymbol{c}_{m}, \boldsymbol{c}_{n} \in \mathbf{C}_{T}$. The selection of $\sigma$ and the bandwidth $B W$ in the Gaussian weight function are discussed in previous subsection.

3. Find the sum of all the weighted intersections as:

$$
\eta \leftarrow \eta+\gamma
$$

The final $\eta$ is exported as the matching rate between two $\mathrm{CCH}$ histograms. Since both histograms have been normalised to $[0,1]$, the resultant $\eta$ takes value in the range of $[0,1]$. The larger the matching rate is, the higher similarity the two images have, when the $\mathrm{CCH}$ histograms are concerned.

Note that in above procedure, the larger distance of $d_{1}$ and $d_{2}$ is taken to decide the weight. The reason for this is that for any two pairs of colours in two images respectively, as long as there is one pair of colours being viewed as "dissimilar", the CCH between these two pairs of colours should not be matched. This design further guarantees that the intersection between $\mathrm{CCH}$ histogram bins only happens on "similar" colour pairs with the condition that both colour pairs must be viewed as "similar" before an intersection is computed.

\section{Experiments and Discussion}

\subsection{Selection of Model and Parameters}

As mentioned earlier in this paper, a model $\mathrm{CCH}$ histogram is needed to detect regions of interest. The selection of the model $\mathrm{CCH}$ can take either of the following two ways. According to our experimental results, there is not significant difference in the detection results.

The first way is a straightforward method. From the training data, i.e., the images of sample swimming pools, manually pick up one image which colour intensities are perceptually not too bright nor too dark compared with other training sample images. For instance, we have tried using the sixth sample in the first row of Figure 2 as the model image. The $\mathrm{CCH}$ histogram of the model image is then computed as the model $\mathrm{CCH}$, and this histogram is used to compare with $\mathrm{CCH}$ histograms computed at each position while the scanning window is 
moving around the image. Regions that give a higher matching score are kept as ROIs.

The second method is based on statistical analysis on the training data. For each training sample, the $\mathrm{CCH}$ histogram is firstly computed. Then, a single statistical average $\mathrm{CCH}$ histogram is obtained based on all of the $\mathrm{CCH}$ histograms of training samples. This average histogram is used as the model CCH histogram. Same searching procedure as in the first method is performed to find the ROIs.

The size of the scanning window in this algorithm is selected considering the minimum size of possible swimming pools in images that the algorithm is able to detect. In our experiments, the scanning window is a $8 \times 8$-pixel square window. The moving step is normally set as half of the width and half of the height of the scanning window, i.e., 4-pixel. However, a finer ROI result can be obtained with smaller step value at the cost of more iterations and hence longer searching time.

Another very important parameter is used to decide how similar a region's $\mathrm{CCH}$ is to the model $\mathrm{CCH}$ so that the region can be taken as a region of candidate. Again, this can be decided via statistical analysis on the training samples. First, the matching score of each training sample to the model in terms of $\mathrm{CCH}$ histogram is computed. Then, the minimum value of the matching scores can be set as the threshold of deciding whether a region can be taken as a candidate. In our experiments, regions that have a matching score with the model $\mathrm{CCH}$ histogram larger than 0.5 are reserved as ROIs.

\subsection{Experimental Results}

In this algorithm, we first use the $\mathrm{CCH}$ histogram to search ROIs. Then, the segmented regions based on the colour and edge information which are covered by ROIs are returned as the detection result. One of our experimental results is shown in Figure 1.

Figure 4(a) shows another example of a partial aerial image. Figure 4(b) shows the image segmentation result using a mean shift filter, where different regions are painted in different colours. Figure $4(\mathrm{c})$ is the detected ROIs via comparing the $\mathrm{CCH}$ histograms of regions covered by scanning window and the model $\mathrm{CCH}$, where the background are marked as black points. The final detected swimming pools are shown in Figure 4(d), where finer detection results are retrieved from Figure 4(b) which are covered by ROIs in Figure 4(c).

\section{Conclusions and Future Work}

In this paper, we present the idea of using local cooccurring patterns for object detection from aerial images. For this application, we propose to use the cooccurrence patterns of local features, including colour features and edge-based shape features, for object detection purpose. A semi-automatic method to detect any object from aerial images is presented. In this method, the users can use the 


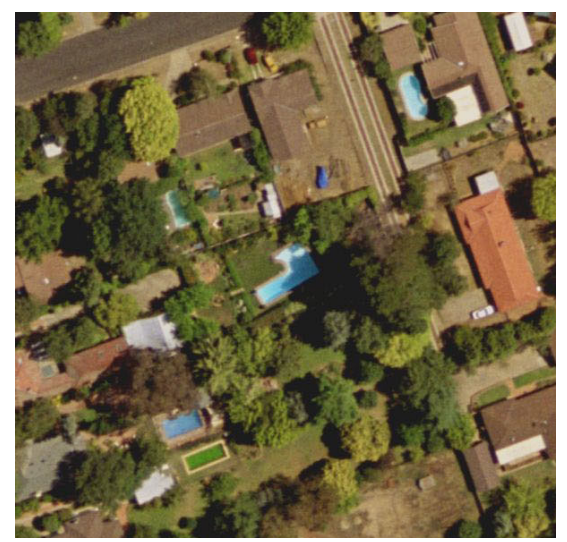

(a) Original image

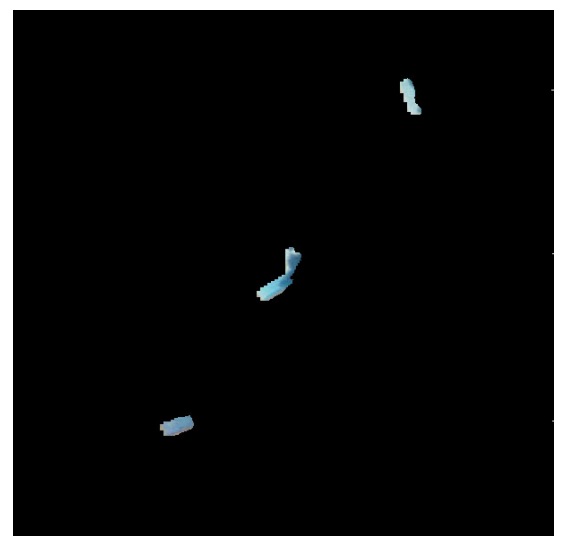

(c) Detected ROIs

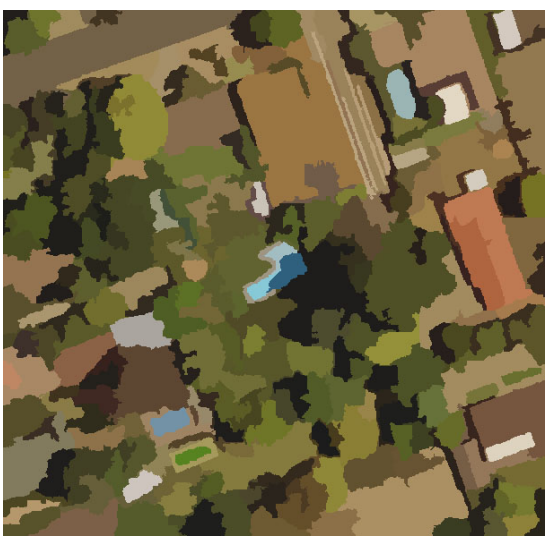

(b) Mean shift segmented

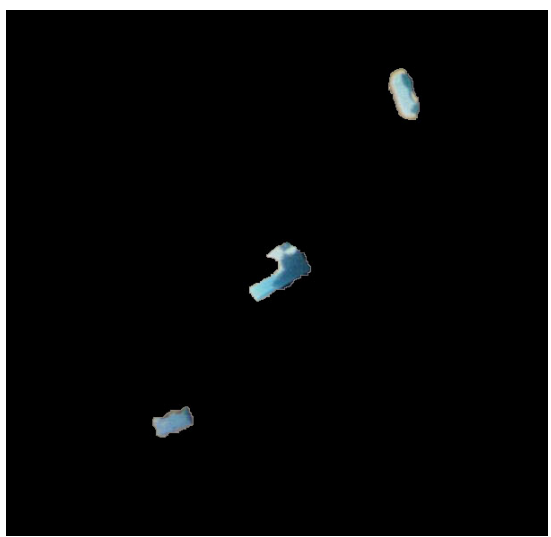

(d) Final detection result

Fig. 4. Applying local cooccurring patterns for swimming pool detection.

mouse to click any objects of interest, the systems then automatically computes and picks up an area and uses this as a training set.

Our future work in the next step is to study more complex shape features and combine the cooccurring patterns associated with the colour-based features to train a classifier for detecting randomly selected objects.

Acknowledgments. The work has been supported by the Cooperative Research Centre for Spatial Information, whose activities are funded by the Australian Commonwealth's Cooperative Research Centres Programme.

\section{References}

1. Mena, J.B.: State of the art on automatic road extraction for gis update: a novel classification. Pattern Recognition Letters 24(16) (2003) 3037-3058 
2. Wang, H., Miller, P.: Disvoering the local co-occurring pattern in visual categorization. Proceedings of the IEEE International Converence on Advanced Video and Signal based Surveillance Ssystem (2006)

3. Mena, J., Malpica, J.: An automatic method for road extraction in rural and semiurban areas starting from high resolution satellite imagery. Pattern Recognition Letters 26(9) (2005) 1201-1220

4. Mena, J., Malpica, J.: Color image segmentation using the dempster-shafter theory of evidence for the fusion of texture. Proceedings of the ISPRS Workshop XXXIV3/W8 (2003) 139-144

5. Mena, J.B., Malpica, J.A.: Color image segmentation based on three levels of texture statistical evaluation. Applied Mathematics and Computation 161(1) (2005) $1-17$

6. Jia, W., Tien, D.: Discovering local cooccurring patterns from aerial images. Proceedings of the International Conference on Information Technology and Applications (2007) 300-305

7. Chang, P., Krumm, J.: Object recognition with color cooccurrence histograms. Proceedings of the 1999 IEEE Computer Society Conference on Computer Vision and Pattern Recognition 2 (1999) 498-504

8. Jia, W., Zhang, H., He, X., Wu, Q.: Image matching using colour edge cooccurrence histograms. Proceedings of the 2006 IEEE International Conference on Systems, Man, and Cybernetics (SMC06) (2006) 2413-249

9. Wu, X.: Efficient statistical computations for optimal color quantization. Graphics Gems 2 (1991) 126-133

10. Crandall, D., Luo, J.: Robust color object detection using spatial-color joint probability functions. Proceedings of the 2004 IEEE Computer Society Conference on Computer Vision and Pattern Recognition 1 (2004) 379-385

11. Kelly, K.L., Judd, D.B.: Color universal language and dictionary of names. National Bureau of Standards special publication 440. Washington, DC: U.S. Department of Commerce, National Bureau of Standards (1976)

12. Giorgianni, E.J., Madden, T.E.: Digital Color Management: Encoding Solutions. Addison-Wesley, Reading, MA (1997)

13. Tomasi, C., Manduchi, R.: Bilateral filtering for gray and color images. Proceedings of the Sixth International Conference on Computer Vision (1998) 839-846

14. Paris, S., Durand, F.: A fast approximation of the bilateral filter using a signal processing approach. Proceedings of the European Conference on Computer Vision (2006)

15. Comaniciu, D., Meer, P.: Mean shift analysis and applications. Proceedings of the Seventh IEEE International Conference on Computer Vision 2 (1999) 1197-1203

16. Wong, K., Cheung, C., Po, L.: Merged-color histogram for color image retrieval. Proceedings of the International Conference on Image Processing 3 (2002) 949-952

17. Jia, W., Zhang, H., He, X., Wu, Q.: Gaussian weighted histogram intersection for license plates classification. Proceedings of the 18th International Conference on Pattern Recognition (2006) 574-577

18. Jia, W., Zhang, H., He, X., Wu, Q.: Refined gaussian weighted histogram intersection and its application in number plate categorization. Proceedings of the 3rd International Conference on Computer Graphics, Imaging and Visualization (2006) $249-254$ 